PACS: 71.25.Rk, 81.60Cp

\title{
Photoelectrical characteristics of two-dimensional macroporous silicon structures
}

\author{
L.A. Karachevtseva, V.F. Onischenko, M.I. Karas', O.I. Dandur'yants, F.F. Sizov, O.J. Stronska \\ V. Lashkaryov Institute of Semiconductor Physics, NAS of Ukraine, 41, prospect Nauky, 03028 Kyiv, Ukraine \\ Phone: 38 (044) 525-98-15. Fax: 38 (044) 525-83-42 \\ E-mail:lakar@isp.kiev.ua; sizov@isp.kiev.ua; stronska@isp.kiev.ua
}

\begin{abstract}
Photoelectrical properties of macroporous silicon structures were investigated in the near infrared spectral range $(1$ to $8 \mu \mathrm{m})$. Angular dependences of photoconductivity, its amplification, realization of the single-mode optical regime, essential domination of the absorption over the light reflection by structures of macroporous silicon are explained by formation of the plasmon type surface polaritons. Photoconductivity bands correlate with maxima of intrinsic and impurity light absorption. The change in the photoconductivity value is mainly determined by the growth of the electron mobility.
\end{abstract}

Keywords: macroporous silicon structures, optical transmittance, photoelectrical characteristics.

Manuscript received 14.10.04; accepted for publication 16.12.04.

\section{Introduction}

The study of photonic crystals are a new direction of solid-state physics that is rapidly progressing. These crystals are periodic structures with periodic modulation of permittivity where the photonic bands are formed by analogy to electronic bands in crystals. The perspective material for two-dimensional photonic structures is the macroporous silicon obtained by a method of photoanodic etching. It is promoted by low-cost technology of electrochemical etching, possibility to create structures with a necessary geometry, the high ratio of the pore depth to the pore diameter. The macroporous silicon structures are perspective for the development of optical devices and sensors in the near infrared spectral range due to the presence of intensive absorption bands [1-3]. This paper concerns the results of studying the photoelectrical properties of macroporous silicon in near infrared spectral range $(1 \ldots 8 \mu \mathrm{m})$. In particular, studied were the angular dependences of photoconductivity, optical transmittance, photoconductivity spectra, and relaxation time. Dependences of electron conductivity, concentration and mobility on the illumination intensity were also researched.

\section{Experiment}

The initial samples were $n$-type silicon with (100) orientation and 2 to $5 \mathrm{Ohm} \cdot \mathrm{cm}$ conductivity. Macropores of 1 to $15 \mu \mathrm{m}$ diameter were formed due to generation and transfer of nonequilibrium holes to the electrochemically treated $n$-Si surface after the optical band-to-band electron-hole generation $[1,2]$. Initial $n$-Si plates were chemically polished in 1:3 $\mathrm{HF}$ and $\mathrm{HNO}_{3}$ solutions and anisotropically etched in $10 \%$ solution of $\mathrm{KOH}$. An ohmic contact was fabricated by rubbing InGa eutectic alloy around the circular area exposed to the electrolyte. The samples were mounted in an electrochemical cell and connected with the potentiostat by the three-electrode scheme. The electrolyte was 5 weight $\%$ solution of hydrofluoric acid. The applied voltage was measured in relation to a platinum wire near the sample surface. During the electrochemical etching process, the samples were illuminated by radiation of $100 \mathrm{~W}$ tungsten lamp. Periodic structures as well as structures with arbitrary distribution of macropores have been fabricated (Fig. 1).

Optical transmittance was measured using IR Fourier spectrometer IFS-113, IR spectrophotometer, He-Ne laser and Specord M85.

\section{Results and discussion}

The photoconductivity of macroporous silicon structures was measured. It depends on the direction of electromagnetic radiation (Fig. 2). There are maxima of photoconductivity which are observed at the normal incidence of electromagnetic radiation, at angles close to the total internal reflection angle relatively to macropore 

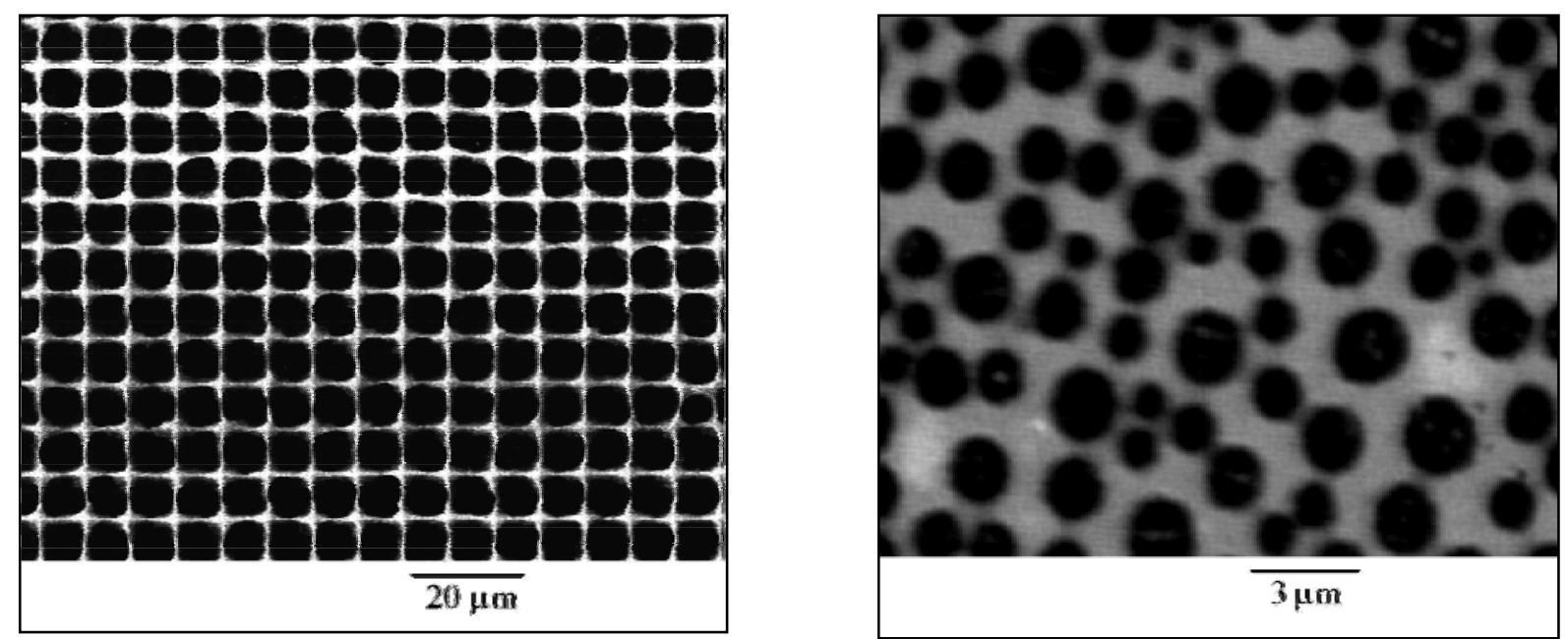

Fig. 1. Microphotos of macroporous silicon structures with periodic and arbitrary distributed pores.

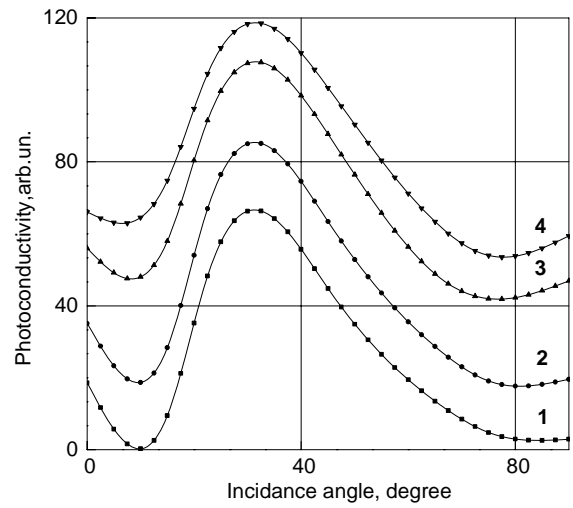

Fig. 2. Photoconductivity dependence of macroporous silicon structures on the angle of incidence for the electromagnetic radiation $(\lambda=0.94 \mu \mathrm{m})$ at the voltage (in $\mathrm{mV}$ ) on the sample without illumination: $1-3 ; 2-27 ; 3-$ $55 ; 4-70(T=77 \mathrm{~K})$.

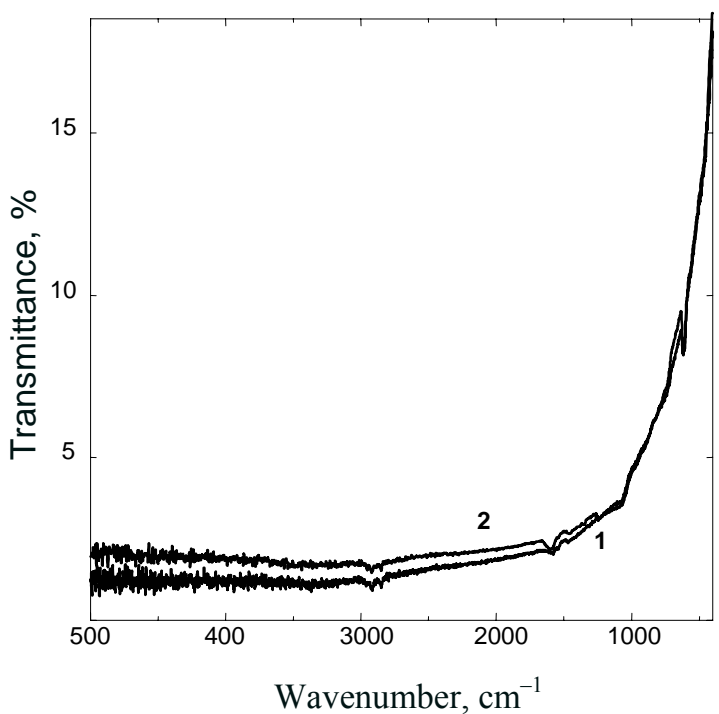

Fig. 3. Transmittance spectra of two macroporous silicon structures.

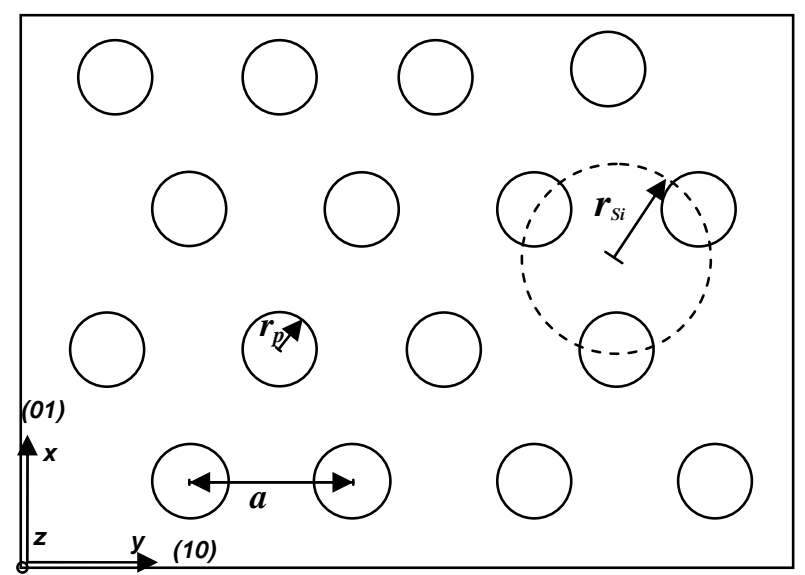

Fig. 4. Scheme of periodic macroporous silicon structure with the pore radius $r_{p}$ and the radius of a silicon column $r_{\mathrm{Si}}$.

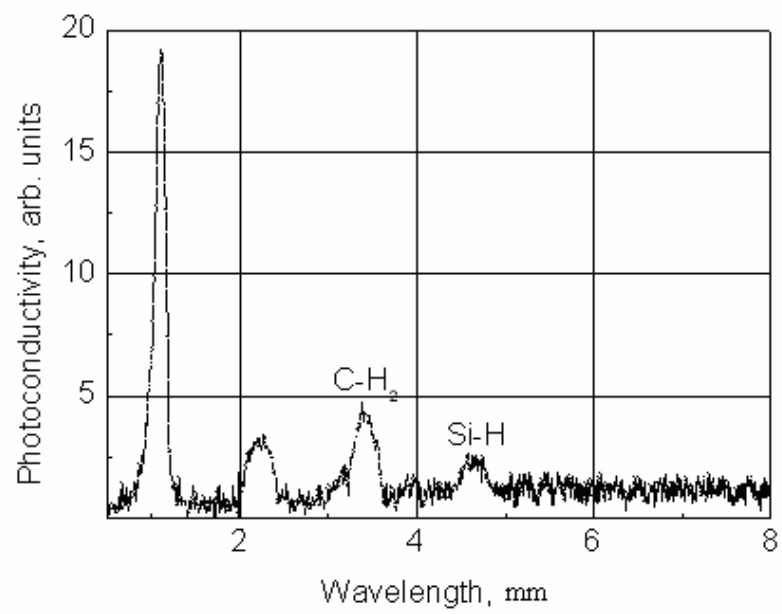

Fig. 5. Photoconductivity spectral dependence of the macroporous silicon structure.

(C) 2004, V. Lashkaryov Institute of Semiconductor Physics, National Academy of Sciences of Ukraine 


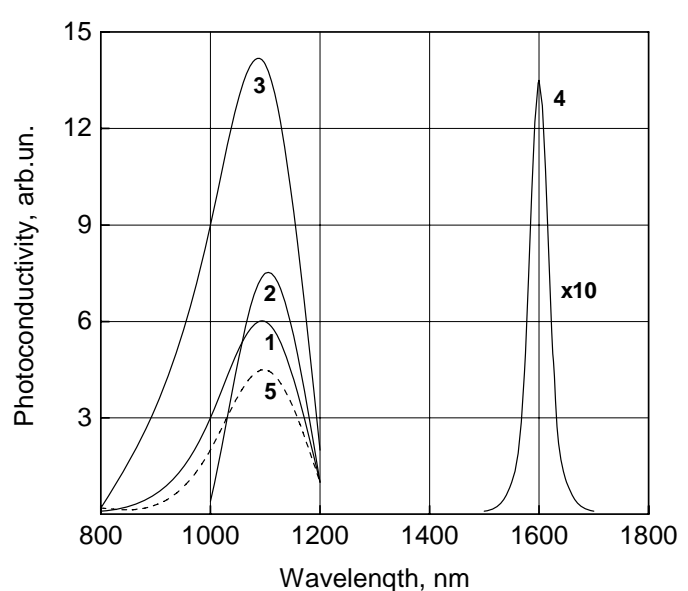

Fig. 6a. Photoconductivity spectral dependence for silicon single crystal (5) and macroporous silicon structures in the range of the intrinsic light absorption (1-4).

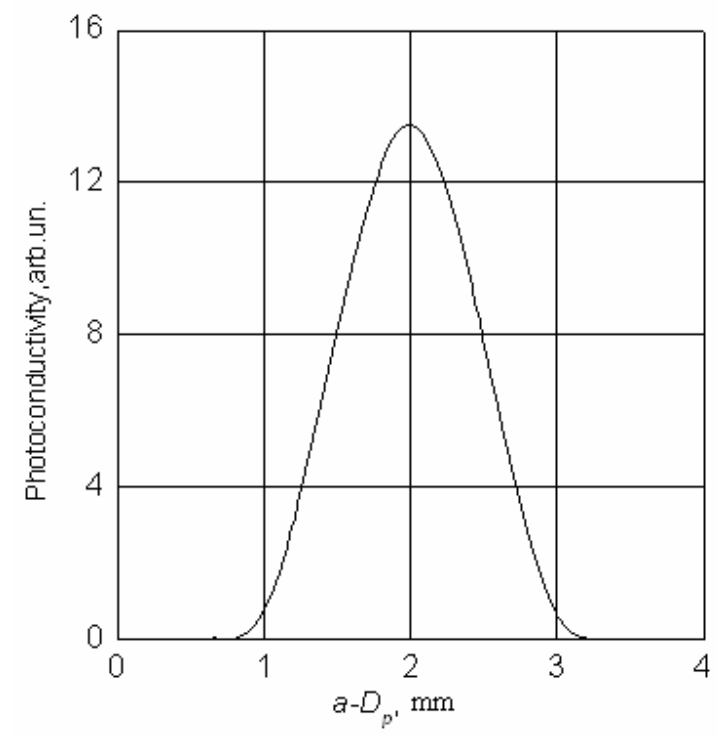

Fig. 6b. Photoconductivity signal maximum dependence on the distance between macropores.

walls, and at grazing light incidence relatively to the structure surface. Such dependence of photoconductivity is caused by the optical characteristics.

The optical transmittance coefficient of macroporous silicon structures is more than that for the homogeneous material by a factor of $10^{2}$ in the range of wavelengths smaller than the optical period of macroporous structure (Fig. 3). Absorption coefficient is in the range from 300 to $500 \mathrm{~cm}^{-1}$. Absorption peaks and steps are observed in the spectrum of impurity absorption. In the longwave region of the spectrum, the step frequency is proportional to the distance between

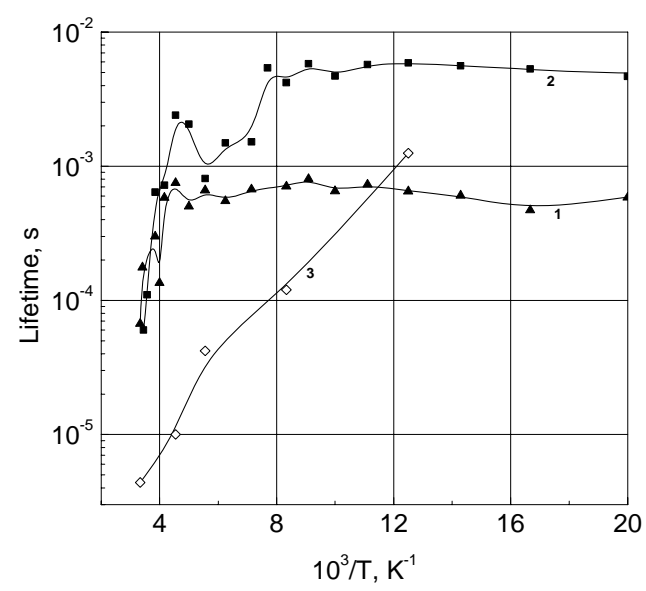

Fig. 7. Temperature dependences of the relaxation time of photoconductivity for two samples of: macroporous $(1,2)$ and monocrystalline (3) silicon.

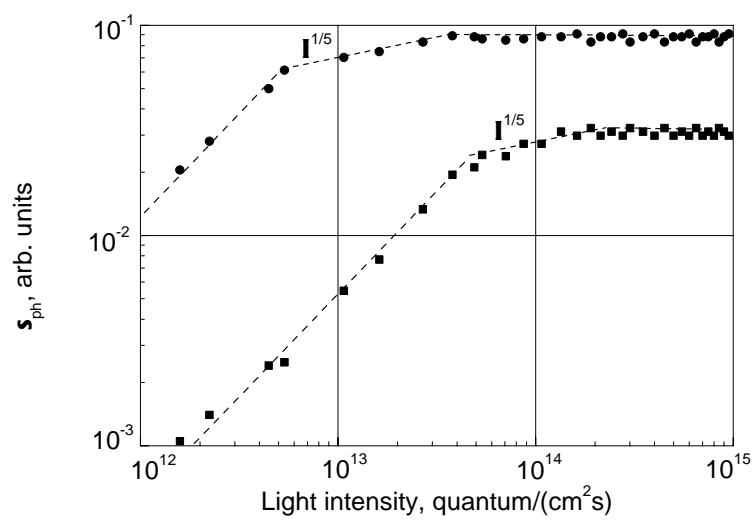

Fig. 8. Photoconductivity dependence on the illumination intensity for macroporous silicon structures $300 \mathrm{~K})$.

macropores and, in the shortwave region, it is proportional to the diameter of macropores. In the previous publication [3], it was shown that, on macroporous silicon structure, the optical modes are formed on silicon columns in longwave region and on the macropores in the shortwave one (Fig. 4). So, the photoconductivity has been measured in this spectral range, where the directed optical modes are formed on macropores.

The transmission spectra of macroporous silicon structures were measured by a spectrophotometer with the aperture of about $10^{\circ}$, therefore, at the formation of the 
optical mode, the multimode regime should be realized. However, the step formation testifies to realization of the single mode regime, which is caused by the surface oscillator fluctuations on a macropore surface and surface polariton formation. This is supported by the preferable absorption of the $p$-components of the incident electromagnetic radiation by macroporous silicon structures. Besides, we measured the reduction in the intensity of reflected light and increase in the absorption. It is an additional feature of exciting the surface electromagnetic waves $[4,5]$.

In other words, at the normal light incidence, the directed optical modes are formed by macropores. Surface waves are formed in the region of the angle of total internal reflection respectively to macropore walls. At grazing incidence, the surface diffraction that is caused by the surface periodic relief occurs. Thus, the optical modes interact with a surface and excite the surface polaritons [6].

On the plots of the photoconductivity dependence on the angle of the electromagnetic radiation incidence, we observed the maxima in the case of the normal incidence in the region of the angle of the total internal reflection respectively to macropore walls, and at a grazing angle of incidence respectively to the structure surface.

Franz-Keldysh oscillations were registered in electroreflectance spectra of the macroporous silicon structures [7]. The oscillation period corresponds to the built-in electric field of $(5 \ldots 9) \cdot 10^{5} \mathrm{~V} / \mathrm{cm}$. It is caused by the surface conditions at the interface silicon - oxide silicon, which generate the two-dimensional electron gas enriched layer of 0.6 to $1 \mathrm{~nm}$ thickness.

It is established that maxima of photoconductivity are observed in the field of intrinsic absorption, on a surface bonds, $\mathrm{C}-\mathrm{H}, \mathrm{Si}-\mathrm{H}$ bonds, generated on a surface after electrochemical etching (Fig. 5). Thus, the photoconductivity is determined by generation of photocarriers at the surface in the enriched layer. Correspondence of the photoconductivity spectra of macroporous silicon structures and those of monocrystal silicon testifies to the enrichment of a macropore surface by photocarriers and formation of the plasmon type surface polaritons in this spectral region $[4,6]$.

The enhancement of the photoconductivity is shown in Fig. 6a where the spectral dependence of photoconductivity is presented in the region of band-toband light absorption of monocrystalline silicon and structures of macroporous silicon. It can be seen from Fig. $6 a$ that, in the macroporous silicon structures, the signal of photoconductivity is higher by a factor of 30 than that for a single crystal substrate. At the maximal enhancement of photoconductivity (Fig. 6a, curve 4), the peak shift into the longwave region is measured. The dependence of maximum of photoconductivity spectrum signal on the distance between macropores $a-D_{p}$ is presented in Fig. $6 \mathrm{~b}$. The greatest value of the photoconductivity signal is measured at the distance between macropores $a-D_{p}=2 \mu \mathrm{m}$.

The photoconductivity relaxation time of macroporous silicon structures comprises values 20 to $80 \mu$ s at $T=300 \mathrm{~K}$, which is by the order larger than the relaxation time for monocrystalline silicon (Fig. 7).

The dependence of electronic conductivity on the intensity of illumination for macroporous silicon structures measured at $0.9 \mu \mathrm{m}$ wavelength is shown in (Fig. 8). There were revealed the linear dependence of photoconductivity at the intensity of illumination within the range $2 \cdot 10^{12}$ to $4 \cdot 10^{12}$ quantum $/\left(\mathrm{cm}^{2} \cdot \mathrm{s}\right)$, the power dependence with an exponent $1 / 5$ within the range of intensities $5 \cdot 10^{12}$ to $4 \cdot 10^{13}$ quantum $/\left(\mathrm{cm}^{2} \cdot \mathrm{s}\right)$, and the saturation within the range $5 \cdot 10^{13}$ to $3 \cdot 10^{14}$ quantum $/\left(\mathrm{cm}^{2} \cdot \mathrm{s}\right)$ at $T=78 \mathrm{~K}$. The power dependence with an exponent $1 / 5$ is classical [8]. It shows the increase in surface potential due to increasing the concentration of photocarriers for macropore surfaces. In this case, the increase in the surface potential can be caused by the formation of surface polaritons. Thus, the significant charge of nonequilibrium carriers can be collected as a result of decreasing the surface recombination rate. The measured photoresponse is increased with the intensity of illumination almost by the order of its values at room temperatures (Fig. 8). It is the result of accelerated photocarrier recombination. The Hall measurements showed that the growth of conductivity at linear and power parts of the dependences on the light intensity is caused by the increased electron mobility.

The objects of the further researches are theoretical estimations of processes in the field of a spatial charge (1), the contribution of surface levels and electronic gas of the enriched layer into the magnitude of photoconductivity (2), and peculiarities of electron scattering by the macropore surface potential in connection with the surface polariton formation.

\section{Conclusions}

Photoconductivity is measured in structures of macroporous silicon. There are maxima of photoconductivity observed at the normal incidence of the electromagnetic radiation, at angles close to the total internal reflection angle relatively to macropore walls, and at grazing light incidence relatively to the surface of the structure.

The angular dependences of photoconductivity, its amplification, formation of optical modes and realization of a single-mode regime, essential exceeding the absorption over reflection of light by structures of macroporous silicon are caused by the interaction of optical modes with amplitude fluctuations of oscillators at the macropore surface and with the surface polariton formation.

The photoconductivity bands correlate with maxima of intrinsic and impurity light absorption. The absolute 
maximum of photoconductivity is observed when the distance between macropores $a-D_{p}=2 \mu \mathrm{m}$. Photoconductivity is mainly determined by the growth of the electron mobility.

\section{References}

1. L.A. Karachevtseva, O.A. Litvinenko, E.A. 6 Malovichko, Stabilization of electrochemical formation of macropores in $n$-Si // Journ. of Theor. and Experim. Chem. 34 (5), p. 314-318 (1998).

2. L.A. Karachevtseva, O.A. Lytvynenko, O.J. Stronska, Development and optical characteristics of the macroporous silicon structures // Semiconductor Physics, Quantum Electronics \& Optoelectronics, 3 (1), p. 22-25 (2000).

3. L.A. Karachevtseva, O.A. Litvinenko, E.A. Malovichko, O.J. Stronska, Optical transmittance of 2D macroporous silicon structures // Ibid. 4 (4), p. 347-351 (2001).
4. M.I. Dmitruk, V.G. Litovchenko, V.L. Strizshevsky, Surface polaritons in semiconductors and dielectrics, Naukova Dumka, Kyiv (1989) (in Russian).

5. E.F. Venger, A.V. Goncharenko, M.I. Dmitruk, Optics of small particles and disperse media, Naukova Dumka, Kyiv (1999) (in Russian).

L.A. Karachevtseva, M.I. Karas', V.F. Onishchenko, F.F. Sizov, Enhancement of the photoconductivity in 2D photonic macroporous silicon structures // Photonic Crystal Materials and Devices II, Proc. SPIE, 5360, p. 381-389 (2004).

7. R.Yu. Holiney, L.A. Matveeva, E.F. Venger, O.A. Litvinenko, L.A. Karachevtseva, Electroreflectance study of macroporous silicon structures // Appl. Surf. Sci. 172 (3), p. 214-219 (2001).

8. A.V. Sachenko, O.V. Snitko, Photoeffects in presurface semiconductor layers, Naukova Dumka, Kyiv (1984) (in Russian). 